TRANSACTIONS OF THE

AMERICAN MATHEMATICAL SOCIETY

Volume 358, Number 4, Pages 1781-1796

S 0002-9947(05)03779-7

Article electronically published on October 21, 2005

\title{
AUTOMORPHISMS OF COXETER GROUPS
}

\author{
PATRICK BAHLS
}

\begin{abstract}
We compute $\operatorname{Aut}(W)$ for any even Coxeter group whose Coxeter diagram is connected, contains no edges labeled 2, and cannot be separated into more than 2 connected components by removing a single vertex. The description is given explicitly in terms of the given presentation for the Coxeter group and admits an easy characterization of those groups $W$ for which $\operatorname{Out}(W)$ is finite.
\end{abstract}

\section{INTRODUCTION}

A Coxeter system is a pair $(W, S)$ consisting of a group $W$ with a distinguished generating set $S=\left\{s_{i}\right\}_{i \in I}$ for which there is a presentation of the form $\langle S \mid R\rangle$, where

$$
R=\left\{\left(s_{i} s_{j}\right)^{m_{i j}} \mid m_{i j} \in\{1,2, \ldots, \infty\}, m_{i j}=1 \Leftrightarrow i=j, \text { and } m_{i j}=m_{j i}\right\} .
$$

We call $W$ a Coxeter group if it possesses a generating set $S$ for which $(W, S)$ is a Coxeter system. Such a set is called a fundamental generating set for $W$. If one may choose such a set $S$ so that all of the exponents $m_{i j}$ (for $i \neq j$ ) are either even or infinite, $W$ is said to be even, and $(W, S)$ is an even system. The main result of [1] shows that for a given Coxeter group $W$, this even system is essentially unique.

We now introduce a graph-theoretical representation of a Coxeter system $(W, S)$. The Coxeter diagram $\mathcal{V}$ corresponding to the system $(W, S)$ is an edge-labeled graph whose vertex set is in one-to-one correspondence with the set $S$ and for which there is an edge labeled $m_{i j}$ between vertices $s_{1}$ and $s_{2}$ if and only if $m_{i j}<\infty$. It is clear that the diagram $\mathcal{V}$ is completely determined by the system $(W, S)$ and vice versa. We will frequently let $\mathcal{V}$ denote the vertex set of the Coxeter diagram; this abuse should not cause confusion in context.

We fix the above notation throughout the remainder of this paper, so that $\mathcal{V}$ will always refer to the diagram corresponding to the system $(W, S)$.

In this paper we will concern ourselves first with Coxeter groups whose diagrams are connected. (Note that if $\mathcal{V}$ is not connected, then $W$ can be represented as a free product of the groups generated by the individual components of the diagram $\mathcal{V}$. More will be said about this in Section 6.)

We call the Coxeter group $W$ rigid if given any two systems $\left(W, S_{1}\right)$ and $\left(W, S_{2}\right)$, there is an automorphism $\alpha \in \operatorname{Aut}(W)$ such that $\alpha\left(S_{1}\right)=S_{2}$. If such an automorphism can always be chosen from $\operatorname{Inn}(W)$, the group of inner automorphisms of $W$,

Received by the editors May 20, 2003 and, in revised form, July 9, 2004.

2000 Mathematics Subject Classification. Primary 20F28, 20 F55.

Key words and phrases. Coxeter group, group automorphism.

The author was supported by an NSF VIGRE postdoctoral grant.

(C)2005 American Mathematical Society Reverts to public domain 28 years from publication 
then $W$ is called strongly rigid. (In both of these cases, any two diagrams $\mathcal{V}_{1}$ and $\mathcal{V}_{2}$ for $W$ are isomorphic as edge-labeled graphs.) In case $W$ is infinite and strongly rigid, the group $\operatorname{Aut}(W)$ has a very simple structure (see [6]):

$$
\operatorname{Aut}(W) \cong \operatorname{Inn}(W) \times \operatorname{Diag}(W)
$$

where $\operatorname{Diag}(W)$ consists of the diagram automorphisms of $W$, those which are induced (in the obvious fashion) by symmetries of the unique diagram $\mathcal{V}$ corresponding to $W$. (When $W$ is finite, this formula may not be true, because $\operatorname{Diag}(W)$ may lie inside $\operatorname{Inn}(W)$, as is the case with the dihedral groups $D_{k}$ for $k$ odd, and the symmetric group $\Sigma_{k}$ on $k$ letters. Since our concern is primarily with infinite Coxeter groups, we will not concern ourselves with these groups.)

The goal of this paper is to describe the automorphism group $\operatorname{Aut}(W)$ of a given even Coxeter group $W$ which satisfies weaker conditions than strong rigidity. This description will admit a finite presentation and will naturally generalize the description given above.

Given a Coxeter system $(W, S)$, any element of the form $w s w^{-1}$ where $w \in W$ and $s \in S$ is called a reflection of the system $(W, S)$. (This terminology stems from the geometric action of such Coxeter group elements as reflections across hyperplanes in some linear space.) If, for the group $W$, any two systems $\left(W, S_{1}\right)$ and $\left(W, S_{2}\right)$ yield the same set of reflections, we call $W$ reflection independent. It is clear that strong rigidity implies reflection independence. More interestingly, if $W$ is even and reflection independent, then it is rigid (see [3]).

We say that a Coxeter system is of large type if the corresponding diagram has no edges labeled 2. Using the main theorem of [3] we conclude that any large-type even Coxeter group is almost always reflection independent, and therefore rigid. These are the groups with which we shall be concerned. (The only obstacle to reflection independence is the presence of "spikes" with label $2(2 k+1), k \geq 1$; these are edges $[s t]$ in which one of the vertices, say $s$, has valence 1 in the diagram.)

In 2, necessary conditions for an even Coxeter group to be strongly rigid were given, and these conditions were shown to be sufficient provided that $W$ is reflection independent and that either $\mathcal{V}$ has no simple circuits of length less than 5 (i.e., no "triangles" and no "squares") or $W$ is of large type.

We will mimic the method of proof used in that paper in order to compute $\operatorname{Aut}(W)$ for large-type even groups which additionally satisfy the following condition:

$(\mathrm{NVB}) \mathcal{V}$ contains no vertex $s$ so that $\mathcal{V} \backslash\{s\}$ consists of more than 2 connected components.

If $\mathcal{V}$ satisfies this condition, we shall say that it has no vertex branching, or is NVB. We shall also say that $W$ is NVB if its diagram is NVB.

Theorem 1.1. Let $W$ be an even, large-type, NVB Coxeter group with connected diagram $\mathcal{V}$. Then $\operatorname{Aut}(W)$ is a semidirect product of $G$ with $\operatorname{Diag}(W)$, where, up to a subgroup of finite index, $G$ is a product of $\operatorname{Inn}(W)$ with certain subgroups of centralizers of edges and vertices of the diagram $\mathcal{V}$.

Remarks. The group $G$ which appears in Theorem 1.1 is none other than the group $\operatorname{Spe}(W)$ of special automorphisms of $W$, as defined, for instance, in [15. Our main theorem shows that $\operatorname{Aut}(W)$ is a semidirect extension of $\operatorname{Spe}(\mathrm{W})$ by $\operatorname{Diag}(W)$, a property also possessed by right-angled Coxeter groups (as shown by Tits in [18]). The precise form of $G$ will be given in Section 5 , once we have developed a bit more 
notation and terminology, although the automorphisms that we construct there can be compared to the notion of a Dehn twist.

A Dehn twist $\phi$ of an amalgamated product $A *_{C} B$ is an automorphism satisfying $\phi(a)=a$ for all $a \in A$ and $\phi(b)=c b c^{-1}$ for all $b \in B$, where $c$ is a fixed central element in $C$. In [17] Rips and Sela prove that for a torsion-free hyperbolic group $G$ the group of automorphisms of $G$ generated by the inner automorphisms and the Dehn twists relative to a given splitting of $G$ is of finite index in the overall automorphism group. Levitt (in [12) goes further, describing $\operatorname{Aut}(W)$ for a large number of groups, including generalized Baumslag-Solitar groups, using graph-ofgroups decompositions.

The approach used here is quite similar, and the results obtained are likewise similar. We will see that the group $G$ in the formula above is also generated by inner automorphisms and Dehn twists relative to the decomposition of $W$ as a certain free product with amalgamation ( $c f$. [14] and [17]). In fact, a slight modification of the unit graph (defined below in Section 5) yields a graph-of-groups decomposition of $W$ giving a splitting which is respected by $\operatorname{Aut}(W)$ in the sense of Section 2 of 12. (Lemma 4.1 plays a fundamental role in proving this). Applying the method of [12] to this splitting of $W$ as a series of free products with amalgamation yields the group $\operatorname{Aut}(W)$, up to finite index.

As a consequence of the structure of $G$ we shall also obtain:

Corollary 1.2. Let $W$ be an even, large-type, NVB Coxeter group with connected diagram. Then $\operatorname{Out}(W)$ is infinite if and only if there is a vertex $s$ in $\mathcal{V}$ such that $\mathcal{V} \backslash\{s\}$ has two connected components and $s$ is adjacent to more than 2 other vertices in $\mathcal{V}$.

In particular, if there are no cut vertices at all, we shall see that $\operatorname{Aut}(W)$ has a particularly nice form:

Corollary 1.3. Let $W$ be an even, large-type, NVB Coxeter group whose connected diagram $\mathcal{V}$ cannot be disconnected by removal of a single vertex. Then

$$
\operatorname{Aut}(W) \cong\left(\operatorname{Inn}(W) \times Z_{2}^{k}\right) \times \operatorname{Diag}(W)
$$

for some number $k$.

Finally, we will be able to describe the structure of $\operatorname{Out}(W)$, even in the case in which this group is infinite:

Corollary 1.4. Let $W$ be an even, large-type, NVB Coxeter group with connected diagram. Then Out $(W)$ contains a subgroup of finite index isomorphic to a direct product of free powers of the cyclic group $Z_{2}$ of order 2 .

Before proceeding, let us remark that a number of papers have been written regarding automorphisms of Coxeter groups. (See [6, 7], 8], 10, 11, 15], 18.) For instance, 7] and [8] are concerned with 3-generated Coxeter groups, and [10] is concerned with Coxeter groups whose diagrams are complete graphs.

In this paper we deal with "larger" Coxeter groups. Using [14] one can characterize the way in which Coxeter groups decompose as free products with amalgamation. The groups with which we will work here turn out to be the large-type even groups which are indecomposable as free products but which decompose in a rather nice fashion as amalgamated products over finite subgroups. (By way of comparison, if 
$\mathcal{V}$ is a complete graph, $W$ cannot be decomposed non-trivially as an amalgamated product.)

There is hope that the results of this paper might be generalized, both to more general classes of Coxeter groups and to other groups which arise as free products (with amalgamation). Indeed, careful application of the results of 2] should yield formulas (more complicated than those contained in this paper) for automorphism groups of a broader class of even Coxeter groups than is considered here.

The author wishes to extend his warmest thanks to Professor Gilbert Levitt for his helpful insights, in particular for drawing comparisons between the present work and Levitt's own work on group automorphisms.

\section{Centralizers and junctions}

Given a subset $J \subseteq S$, we define the group $W_{J} \leq W$ to be the subgroup of $W$ generated by the generators in $J$, subject to the relations of the original presentation which involve only generators from $J$. It is well known (see, for example, 5]) that $\left(W_{J}, J\right)$ is itself a Coxeter system. Clearly this system is even if $(W, S)$ is.

A subgroup $W_{J}$ defined in this manner is called a standard parabolic subgroup of $(W, S)$. Any conjugate $w W_{J} w^{-1}$ (for $w \in W$ ) of such a subgroup is called a parabolic subgroup of $(W, S)$.

In 4 the centralizers of parabolic subgroups of an even group $W$ are described in terms of the presentation corresponding to the given system $(W, S)$. Of particular interest to us are the centralizers of the standard parabolic subgroups whose generators separate the diagram $\mathcal{V}$ into more than one connected component. These centralizers describe the amount of "flexibility" that we have in creating automorphisms of $W$.

Until Section 6 we consider only even large-type Coxeter groups $W$ without spikes (as defined in the previous section) whose diagrams are connected and NVB. These groups are reflection independent and rigid. (Rigidity of $W$ often allows us to omit mention of the set $S$.)

For any subset $J$ of $S$, let $C(J)$ denote the centralizer of $W_{J}$ in $W$. If $J \subseteq S$ satisfies $C(J) \neq\{1\}$ and the full subgraph generated by the vertices $\mathcal{V} \backslash J$ has more than one connected component, we call $J$ a junction for the Coxeter group $W$.

From Theorem 1.1 in 4 , we may prove the following

Proposition 2.1. Let $W_{J}$ be a spherical subgroup of the even large-type Coxeter group $W$. Then $C(J)$ is trivial unless either

a. $J=\left\{s_{i_{1}}\right\}$ (then $C(J)=\left\langle\left\{s_{i_{1}}\right\} \cup\left\{\left(s_{i} s_{i_{1}}\right)^{\frac{m_{i}}{2}-1} s_{i} \mid m_{i}=m_{i_{1} i}<\infty\right\}\right\rangle$ ), or

b. $J=\left\{s_{i_{1}}, s_{i_{2}}\right\}$ and $m=m_{i_{1} i_{2}}<\infty$ (then $\left.C(J)=\left\{1,\left(s_{i_{1}} s_{i_{2}}\right)^{m / 2}\right\}\right)$.

Now if $W$ is reflection independent and $\alpha \in \operatorname{Aut}(W)$, then the image $\alpha(s)$ of any fundamental generator is conjugate to some generator $s^{\prime}: \alpha(s)=w_{s} s^{\prime} w_{s}^{-1}$, some $w_{s} \in W$. From the formula for $\operatorname{Aut}(W)$ when $W$ is strongly rigid, given in the previous section, we see that $w_{s}$ does not depend on $s$ in this case. In general the relationship between $w_{s}$ and $w_{s^{\prime}}$ for $s \neq s^{\prime}$ will be more complicated. In order to compute $\operatorname{Aut}(W)$ it suffices to understand this relationship.

As we shall see, the junctions in the diagram $\mathcal{V}$ effectively divide $\mathcal{V}$ into subsets $S_{1}, \ldots, S_{k}$ of the vertex set $S$ of $\mathcal{V}$ so that $w_{s}=w_{s^{\prime}}$ provided $s, s^{\prime} \in S_{i}$ for some $i$. Moreover, the structure of each junction will provide information about how to obtain one $w_{s_{i}}$ from an "adjacent" $w_{s_{j}}$ : the quotient of two such words will 
essentially lie in the centralizer of the separating junction. Therefore, beginning with a "basepoint" in the diagram $\mathcal{V}$ and an arbitrary conjugating word for that basepoint, we will travel throughout $\mathcal{V}$ and compute the possible conjugating words for each vertex of $\mathcal{V}$, allowing for the possibility that the conjugating word may be modified every time we cross a junction.

\section{Generators which share a common conjugating word}

Before we concern ourselves with crossing junctions, let us first understand those subsets of the generating set $S$ which share a common conjugating word $w$.

We use terminology introduced in [2]. Consider the diagram $\mathcal{V}$. A simple circuit in $\mathcal{V}$ is a closed edge path $C: C=\left\{\left[s_{1} s_{2}\right], \ldots,\left[s_{k-1} s_{k}\right],\left[s_{k} s_{1}\right]\right\}$, where $s_{i} \neq s_{j}$ for $i \neq j$.

We say that a circuit $C=\left\{\left[s_{1} s_{2}\right], \ldots,\left[s_{k} s_{1}\right]\right\}$ in $\mathcal{V}$ is regular if for any two vertices $s_{i}$ and $s_{j}$ such that $|i-j|>1, s_{i}$ and $s_{j}$ are not adjacent in $\mathcal{V}$. Regular circuits are in some sense "minimal" in length.

It is essentially shown in 2] that if $W$ is a reflection independent even Coxeter group corresponding to the two diagrams $\mathcal{V}$ and $\mathcal{V}^{\prime}$ and

$$
C=\left\{\left[s_{1} s_{2}\right], \ldots,\left[s_{k} s_{1}\right]\right\}
$$

is a regular circuit in $\mathcal{V}$, then there exists a regular circuit

$$
C^{\prime}=\left\{\left[s_{1}^{\prime} s_{2}^{\prime}\right], \ldots,\left[s_{k}^{\prime} s_{1}^{\prime}\right]\right\}
$$

in $\mathcal{V}^{\prime}$ and a single word $w \in W$ such that $s_{i}^{\prime}=w s_{i} w^{-1}$ for all $i=1, \ldots, k$. (In fact, from [1, there exists a graph isomorphism $\gamma: \mathcal{V} \cong \mathcal{V}^{\prime}$ such that $\gamma\left(s_{i}\right)=s_{i}^{\prime}$, for all $i$, so the corresponding circuits are those which are matched up by some graph isomorphism of the diagram $\mathcal{V}$.)

Moreover, if there is sufficient overlap between two such circuits, we can conclude that these two circuits must share a single conjugating word.

Suppose that $C_{1}$ and $C_{2}$ are two regular circuits in $\mathcal{V}$. Let $C_{1}^{\prime}$ and $C_{2}^{\prime}$ be the corresponding circuits in the diagram $\mathcal{V}^{\prime}$, and let $w_{1}$ and $w_{2}$ be the words guaranteed by the previous paragraphs. Let $J$ denote the set of vertices which lie on both cycles. If $J$ does not consist of either a single vertex or two adjacent vertices, Proposition 2.1 shows that $C(J)=\{1\}$. For every vertex $s \in J, w_{1} s w_{1}^{-1}=w_{2} s w_{2}^{-1}$, so that $w_{1}^{-1} w_{2} \in C(s)$. Thus $w_{1}^{-1} w_{2} \in C(J)=\{1\}$, so $w_{1}=w_{2}$.

Furthermore, even if $J$ consists of a single vertex or a pair of adjacent vertices, the arguments from [2] show that $C_{1}$ and $C_{2}$ must share a common conjugating word as long as $J$ is not a junction.

These observations allow us to define a collection $\mathcal{U}$ of subsets of $S$, each of which is maximal with respect to the property that if $s_{i}$ and $s_{j}$ lie in the same subset $U \in \mathcal{U}$, then $s_{i}$ and $s_{j}$ share a common conjugating word. (Although $\mathcal{U}$ will be a cover of $S$, it need not be a partition.)

To construct a typical element of $\mathcal{U}$, begin with a single regular circuit $C$, and let $U(0)=C$. For any other regular circuit $C^{\prime}$ so that $C \cap C^{\prime} \neq \emptyset$ is not a junction, we may place the vertices of $C^{\prime}$ in the same subset, and define $U(1)=U(0) \cup C^{\prime}$. Once we have considered all regular circuits which intersect $C$, we continue by considering those circuits which intersect any $C^{\prime}$ which had been appended to $U(0)$, and so forth, creating a sequence $U(0), U(1), \ldots$ of subsets of $S$. Since the number of regular circuits in a given diagram is finite, this process must terminate with some subset $U(n) \subseteq S$. 
We may apply this process beginning with any point that lies on a regular circuit. However, we may not obtain all of the vertices in $\mathcal{V}$ in this manner, as there may be some vertices which do not lie on a regular circuit. Let $s$ be such a vertex. If $s$ lies on any circuit, it is easy to show that it lies on some regular circuit, so we may assume $s$ does not lie on a circuit, and thus any two vertices to which it is adjacent must lie in different components of the full subgraph on the vertex set $\mathcal{V} \backslash\{s\}$. Since we are assuming that $\mathcal{V}$ is NVB, $s$ must be incident at most 2 edges. The vertex $s$ is a junction if it has degree exceeding 1. Moreover, as will become clear in the following sections, such a vertex $s$ need not share a common conjugating word with any other generator in $S$. Therefore the only set in the collection $\mathcal{U}$ described above which contains this vertex is $\{s\}$.

We will call each of the subsets $U$ described above a unit of the diagram $\mathcal{V}$. It is clear that the method described above yields a unique decomposition of $\mathcal{V}$ into units.

\section{Conjugating Words For ADJaCent units}

We now investigate the relationship between conjugating words for units which are "near" one another in $\mathcal{V}$.

Fix $\phi \in \operatorname{Aut}(W)$. Then $\phi(S)$ is another fundamental generating set for $W$, yielding a diagram $\mathcal{V}^{\prime}$ isomorphic to $\mathcal{V}$. Reflection independence implies that for all $s_{i} \in S$, there is a unique generator $s_{\pi(i)}$ such that $\phi\left(s_{i}\right)$ and $s_{\pi(i)}$ are conjugate to one another. It is easy to check (by using [1] and considering relations of the group $W$ ) that the map $\beta$ defined by $\beta\left(s_{\pi(i)}\right)=s_{i}$ is a diagram automorphism. Therefore by replacing $\phi$ with $\beta \circ \phi$ we can assume that $s_{i}$ and $\phi\left(s_{i}\right)$ are conjugate, for all generators $s_{i}$. We make this assumption throughout this section and the next.

Let $U_{i}$ and $U_{j}$ be units in $\mathcal{U}$ such that either

a. $\quad J=U_{i} \cap U_{j} \neq \emptyset$ is a junction such that $U_{i} \backslash J$ and $U_{j} \backslash J$ are in different connected components of the graph $\mathcal{V} \backslash J$, or

b. there exists a unique edge $\left[s_{i} s_{j}\right]$ for $s_{i} \in U_{i}$ and $s_{j} \in U_{j}$, and removing this edge disconnects the diagram $\mathcal{V}$.

In either case we say that $U_{i}$ and $U_{j}$ are adjacent to one another. Let $w_{i}$ and $w_{j}$ be the conjugating words associated with these units. There are six cases that we now consider:

1. $\left|U_{i}\right|>1,\left|U_{j}\right|>1$, and $\left|U_{i} \cap U_{j}\right|=2$;

2. $\left|U_{i}\right|>1,\left|U_{j}\right|>1$, and $\left|U_{i} \cap U_{j}\right|=1$;

3. $\left|U_{i}\right|>1,\left|U_{j}\right|>1$, and $U_{i} \cap U_{j}=\emptyset$, there is a unique edge $\left[s_{i} s_{j}\right]$ such that $s_{i} \in U_{i}$ and $s_{j} \in U_{j}$, and $\left\{s_{i}\right\},\left\{s_{j}\right\}$ are both single-vertex junctions;

4. $\left|U_{i}\right|>1, U_{j}=\left\{s_{j}\right\}$, and there is a unique edge $\left[s_{i} s_{j}\right]$ such that $s_{i} \in U_{i}$;

5. $U_{i}=\left\{s_{i}\right\},\left|U_{j}\right|>1$, and there is a unique edge $\left[s_{i} s_{j}\right]$ such that $s_{j} \in U_{j}$;

6. $U_{i}=\left\{s_{i}\right\}, U_{j}=\left\{s_{j}\right\}$, and $\left[s_{i} s_{j}\right]$ is an edge in $\mathcal{V}$.

Case 1. In this case both units have at least three vertices (by the results of the previous section) and therefore have trivial centralizers (by Theorem 1.1 of [4]). Let $U_{i} \cap U_{j}=\left\{s_{1}, s_{2}\right\}$ be a junction separating $U_{i}$ and $U_{j}$. Then $|C(J)|=2$, and either $w_{j}=w_{i}$ or $w_{j}=w_{i}\left(s_{1} s_{2}\right)^{\frac{m_{12}}{2}}$.

Case 2. Now we suppose that $U_{i} \cap U_{j}=\{s\}$. By Theorem 1.1 from [4 we may write $w_{i}^{-1} w_{j}$ as a product of $s$ and the words $\left(s_{i} s\right)^{\frac{m_{i}}{2}-1} s_{i}$, where $\left(s_{i} s\right)^{m_{i}}=1$ and each $s_{i}$ is adjacent to $s$ in $\mathcal{V}$. Because $\mathcal{V}$ is NVB, we may partition the vertices 
$s_{i}$ adjacent to $s$ into two disjoints sets, according to the connected component of $\mathcal{V} \backslash\{s\}$ in which $s_{i}$ lies. Suppose that $A_{i}$ and $A_{j}$ are the two connected components of $\mathcal{V} \backslash\{s\}$ which contain $U_{i} \backslash\{s\}$ and $U_{j} \backslash\{s\}$, respectively.

The following lemma is fundamental.

Lemma 4.1. Suppose that $U_{i}$ and $U_{j}$ are separated by a single-vertex junction, as above, and that $w_{i}$ and $w_{j}$ are the respective conjugating words for $U_{i}$ and $U_{j}$. Then $w_{j}=w_{i} \epsilon_{s} u v$, where $\epsilon_{s} \in\{1, s\}, u$ is a (possibly trivial) product of the words $\left(s_{i} s\right)^{\frac{m_{i}}{2}-1} s_{i}$ for $s_{i} \in A_{i}$ adjacent to $s$, and $v$ is a (possibly trivial) product of the words $\left(s_{j} s\right)^{\frac{m_{j}}{2}-1} s_{j}$ for $s_{j} \in A_{j}$ adjacent to $s$.

Thus, the ratio $w_{i}^{-1} w_{j}$ is easily controlled.

In order to prove Lemma 4.1, we make use of the following simple observation, which will be needed in the next section as well.

Lemma 4.2. Suppose that $U$ and $U^{\prime}$ are units of $\mathcal{V}$ which both contain the singlevertex junction $\{s\}$ such that $\left(U \cup U^{\prime}\right) \backslash\{s\}$ lies in a single connected component of $\mathcal{V} \backslash\{s\}$. Then there is a finite sequence of edges $\left[s_{1} s\right],\left[s_{2} s\right], \ldots,\left[s_{m} s\right]$ and a corresponding sequence of units $U=U_{1}, U_{2}, \ldots, U_{m+1}=U^{\prime}$ so that $U_{k} \cap U_{k+1}=$ $\left\{s_{k}, s\right\}$ is a junction separating $U_{k}$ and $U_{k+1}$ for all $k=1, \ldots, m$.

Proof. There is nothing to prove if $U=U^{\prime}$, so we assume this is not the case.

Pick and fix vertices $t \in U$ and $t^{\prime} \in U^{\prime}$ such that $[s t]$ and $\left[s^{\prime} t\right]$ are edges in $\mathcal{V}$. Because $t$ and $t^{\prime}$ lie in the same component of $\mathcal{V} \backslash\{s\}$, we can choose a simple path $p$ which does not contain $s$ and which connects $t$ to $t^{\prime}$. We assume that $p$ has been chosen as the shortest such path. Concatenating this path with the path $\left\{\left[t^{\prime} s\right],[s t]\right\}$, we obtain a circuit $C$.

If $C$ is regular, then $t$ and $t^{\prime}$ lie in a common unit, $U^{\prime \prime}$, which is separated from $U$ by $\{s, t\}$ and from $U^{\prime}$ by $\left\{s, t^{\prime}\right\}$, and we are done.

Otherwise, we can "shorten" $C$ to form a regular circuit (compare [2]). Since $p$ was chosen to be the shortest path from $t$ to $t^{\prime}$ which does not contain $s$, the only way in which $C$ can fail to be regular is if there is some vertex $t^{\prime \prime}$ lying on $p$ for which $\left[s t^{\prime \prime}\right]$ is an edge in $\mathcal{V}$. In this case, we may divide $C$ into two strictly shorter circuits (one containing $\left\{s, t, t^{\prime \prime}\right\}$ and the other $\left\{s, t^{\prime}, t^{\prime \prime}\right\}$ ) and induct on the length of the paths into which $p$ has been subdivided to yield the desired conclusion.

We now prove Lemma 4.1.

Proof. We know that $w_{j}=w_{i} w$ for some word $w \in C(\{s\})$. Let us write $w$ as a product

$$
w=\epsilon_{s} \alpha_{1} \beta_{1} \alpha_{2} \beta_{2} \cdots \alpha_{m} \beta_{m},
$$

where each $\alpha_{k}$ is a product of words $\left(s_{l} s\right)^{\frac{m_{l}}{2}-1} s_{l}, s_{l} \in A_{i}$, and each $\beta_{k}$ is a product of words $\left(s_{l} s\right)^{\frac{m_{l}}{2}-1} s_{l}, s_{l} \in A_{j}$. We can clearly assume that $\alpha_{k} \neq 1$ for $2 \leq k \leq m$ and that $\beta_{k} \neq 1$ for $1 \leq k \leq m-1$.

Let us denote by $\phi$ the automorphism to which the conjugating words $w_{i}$ and $w_{j}$ correspond (that is, in particular, $\phi(t)=w_{i} t w_{i}^{-1}$ for all $t \in U_{i}$, and $\phi(t)=w_{j} t w_{j}^{-1}$ for all $t \in U_{j}$ ). Let $\psi$ denote the map $\phi^{-1}$. We know that there exist words $w_{i}^{\prime}$ and $w_{j}^{\prime}$ such that $\psi(t)=w_{i}^{\prime} t w_{i}^{\prime-1}$ for all $t \in U_{i}$ and $\psi(t)=w_{j}^{\prime} t w_{j}^{\prime-1}$ for all $t \in U_{j}$. Furthermore we can write $w_{j}^{\prime}=w_{i}^{\prime} w^{\prime}$, where $w^{\prime} \in C(\{s\})$, so that $w^{\prime}$ can be written as a product

$$
\epsilon_{s}^{\prime} \alpha_{1}^{\prime} \beta_{1}^{\prime} \cdots \alpha_{n}^{\prime} \beta_{n}^{\prime}
$$


for $\epsilon_{s}^{\prime} \in\{1, s\}$ and words $\alpha_{k}^{\prime}$ and $\beta_{k}^{\prime}$ of forms analogous to those of the words $\alpha_{k}$ and $\beta_{k}$ above.

Consider any vertex $s_{l}$ adjacent to $s$. If $s_{l} \in A_{i}$, Lemma 4.2 shows that there exists a word $\bar{\alpha}_{l}$ which can be written as a product consisting solely of letters from $A_{i} \cup\{s\}$ such that

$$
\psi\left(s_{l}\right)=w_{i}^{\prime} \bar{\alpha}_{l} s_{l} \bar{\alpha}_{l}^{-1} w_{i}^{\prime-1} .
$$

Similarly, if $s_{l} \in A_{j}$, we are guaranteed a word $\bar{\beta}_{l}$ which can be written as a product consisting solely of letters of $A_{j} \cup\{s\}$ such that

$$
\psi\left(s_{l}\right)=w_{j}^{\prime} \bar{\beta}_{l} s_{l} \bar{\beta}_{l}^{-1} w_{j}^{\prime-1} \text {. }
$$

Suppose that $t$ is a vertex in $U_{i}$. Then

$$
t=\psi \circ \phi(t)=\psi\left(w_{i} t w_{i}^{-1}\right)=\psi\left(w_{i}\right) w_{i}^{\prime} t w_{i}^{\prime-1} \psi\left(w_{i}\right)^{-1}
$$

so $\psi\left(w_{i}\right) w_{i}^{\prime}=1$ since $C\left(U_{i}\right)=\{1\}$. Now consider $t \in U_{j}$. Here,

$$
t=\psi \circ \phi(t)=\psi\left(w_{i} w t w^{-1} w_{i}^{-1}\right)=\psi\left(w_{i}\right) \psi(w) w_{i}^{\prime} w^{\prime} t w^{\prime-1} w_{i}^{\prime-1} \psi(w)^{-1} \psi\left(w_{i}\right)^{-1}
$$

so $\psi\left(w_{i}\right) \psi(w) w_{i}^{\prime} w^{\prime}=1$ since $C\left(U_{j}\right)=\{1\}$. But

$$
\psi(w)=\psi\left(\epsilon_{s} \alpha_{1} \beta_{1} \cdots \alpha_{m} \beta_{m}\right)=w_{i}^{\prime} \epsilon_{s} \tilde{\alpha}_{1} w^{\prime} \tilde{\beta}_{1} w^{\prime-1} \tilde{\alpha}_{2} w^{\prime} \tilde{\beta}_{2} w^{\prime-1} \cdots \tilde{\alpha}_{m} w^{\prime} \tilde{\beta}_{m} w_{j}^{\prime-1},
$$

where $\psi\left(\alpha_{k}\right)=w_{i}^{\prime} \tilde{\alpha}_{k} w_{i}^{\prime-1}$ and $\psi\left(\beta_{k}\right)=w_{j}^{\prime} \tilde{\beta}_{k} w_{j}^{\prime-1}$, and equations (1) and (2) guarantee that $\tilde{\alpha}_{k}$ can be written as a product consisting solely of letters in $A_{i} \cup\{s\}$, and $\tilde{\beta}_{k}$ can be written as a product consisting solely of letters of $A_{j} \cup\{s\}$.

Using $\psi\left(w_{i}\right) w_{i}^{\prime}=\psi\left(w_{i}\right) \psi(w) w_{i}^{\prime} w^{\prime}=1$, we obtain

$$
\epsilon_{s} \tilde{\alpha}_{1} w^{\prime} \tilde{\beta}_{1} w^{\prime-1} \cdots \tilde{\alpha}_{m} w^{\prime} \tilde{\beta}_{m}=1 .
$$

Now we expand (3) by writing out $w^{\prime}$ :

$$
\epsilon_{s} \tilde{\alpha}_{1}\left(\epsilon_{s}^{\prime} \alpha_{1}^{\prime} \cdots \beta_{n}^{\prime}\right) \tilde{\beta}_{1}\left(\beta_{n}^{\prime-1} \cdots \alpha_{1}^{\prime-1} \epsilon_{s}^{\prime}\right) \cdots\left(\epsilon_{s}^{\prime} \alpha_{1}^{\prime} \cdots \beta_{n}^{\prime}\right) \tilde{\beta}_{m}=1 .
$$

The occurrences of $\epsilon_{s}^{\prime}$ can be commuted to the front and multiplied with $\epsilon_{s}$ to yield a single word in $\{1, s\}$. What further reduction can be performed? Assuming $w^{\prime}$ has been written in reduced form as a product of the words $\alpha_{k}^{\prime}$ and $\beta_{k}^{\prime}$, the only cancellation that can occur is in one of the following subwords:

a. $\tilde{\alpha}_{1} \alpha_{1}^{\prime}$,

b. $\alpha_{1}^{\prime-1} \tilde{\alpha}_{k} \alpha_{1}^{\prime}, k=2, \ldots, m$,

c. $\beta_{n}^{\prime} \tilde{\beta}_{k} \beta_{n}^{\prime-1}, k=1, \ldots, m-1$, or

d. $\beta_{n}^{\prime} \tilde{\beta_{m}}$.

Consider the second case. If $\alpha_{1}^{\prime-1} \tilde{\alpha}_{k} \alpha_{1}^{\prime}=1$, then $\tilde{\alpha}_{k}=1$, so

$$
\alpha_{k}=\phi\left(w_{i}^{\prime}\right) \tilde{\alpha}_{k} \phi\left(w_{i}^{\prime}\right)^{-1}=1 .
$$

But we have assumed that for $2 \leq k \leq m, \alpha_{k} \neq 1$. Thus the word in the second case above is non-trivial. Similarly we may show that the word in the third case above is non-trivial.

Therefore if $m>1$, what remains after cancellation is a product in words $\left(s_{l} s\right)^{\frac{m_{l}}{2}} s_{l}$ which alternates between blocks of such words for $s_{l} \in A_{i}$ and blocks of such words for $s_{l} \in A_{j}$, and which represents the trivial element. Because the groups that we are considering satisfy the $C^{\prime}\left(\frac{1}{6}\right)$ small cancellation condition, any non-trivial word representing the trivial element must contain more than half of a relator appearing in the symmetrized presentation for the group. (See 13 for more 
details.) However, this is clearly not the case if $m>1$. Therefore $m=1$, and the word $w$ can be written as $\epsilon_{s} u v$ for words $u$ and $v$ described in the statement of the lemma.

(Note that we have assumed that $w$ (resp. $w^{\prime}$ ) begins with some word $\alpha_{1}$ (resp. $\alpha_{1}^{\prime}$ ) and ends with some word $\beta_{m}$ (resp. $\beta_{n}^{\prime}$ ); a moment's thought should convince the reader that the other possibilities are similar.)

Case 3. Here, although $U_{i}$ and $U_{j}$ no longer overlap, there is a bridge $\left[s_{i} s_{j}\right]$ between them in $\mathcal{V}$. (That is, removing this edge disconnects the remaining graph.)

Because the edge $\left[s_{i} s_{j}\right]$ does not lie on a cycle in $\mathcal{V}$, we cannot directly apply the methods of [2]. However, by the assumptions made in the first paragraph of this section, the subgroups $H=W_{\left\{s_{i}, s_{j}\right\}}$ and $W_{\left\{\phi\left(s_{i}\right), \phi\left(s_{j}\right)\right\}}$ are conjugate, so there is a word $w$ satisfying $w s_{i} w^{-1}=\phi\left(s_{i}\right)$ and $w x s_{j} x^{-1} w^{-1}=\phi\left(s_{j}\right)$ for some word $x$ in the letters $s_{i}$ and $s_{j}$ such that $s_{i}$ and $x s_{j} x^{-1}$ generate $H$.

Clearly $w_{i}^{-1} w \in C\left(\left\{s_{i}\right\}\right)$ and $x^{-1} w^{-1} w_{j} \in C\left(\left\{s_{j}\right\}\right)$, so that $w_{i}^{-1} w_{j}=\hat{s}_{i} x \hat{s}_{j}$ for some $\hat{s}_{i} \in C\left(\left\{s_{i}\right\}\right)$ and $\hat{s}_{j} \in C\left(\left\{s_{j}\right\}\right)$. We must understand $x$. Let $n$ be the label on the edge $\left[s_{i} s_{j}\right]$.

Because $s_{i}$ and $x s_{j} x^{-1}$ generate $H, s_{i} x s_{j} x^{-1}=\left(s_{i} s_{j}\right)^{k}$ for some $k$ such that $(k, n)=1$ (where $(k, n)$ denotes the greatest common divisor of $k$ and $n$ ). For a given $k \in\{1, \ldots, n-1\}$ such that $(k, n)=1$, there are four geodesic (that is, shortest among words representing the same group element) words $x$ which satisfy $s_{i} x s_{j} x^{-1}=\left(s_{i} s_{j}\right)^{k}$. Namely, for a given $k, x$ lies in the set

(4) $\quad\left\{x_{1}=\left(s_{j} s_{i}\right)^{\frac{k-1}{2}}, x_{2}=\left(s_{j} s_{i}\right)^{\frac{k-1}{2}} s_{j}, x_{3}=\left(s_{i} s_{j}\right)^{\frac{n-k-1}{2}} s_{i}, x_{4}=\left(s_{i} s_{j}\right)^{\frac{n-k+1}{2}}\right\}$.

Conjugating $s_{j}$ by any one of these elements $x_{l}(k)$ yields the same element of $W$. However, since for the given automorphism $\phi, \phi(s)=w_{j} s w_{j}^{-1}$ must hold for every $s \in U_{j}$, it is clear that different choices of $x_{l}(k)$ for the same $k$ will yield different maps $\phi$.

Suppose that a given automorphism $\phi_{l}$ satisfies

$$
\phi(s)=w_{i} s w_{i}^{-1}
$$

for $s \in U_{i}$ and

$$
\phi(s)=w_{i} u x_{l}(k) v s v^{-1} x_{l}(k)^{-1} v^{-1} w_{i}^{-1}
$$

for $s \in U_{j}$, for some $k, l$, and for some words $u$ and $v$ as described in Case 2 . (That is, $u$ is a product of words $\left(s s_{i}\right)^{\frac{m}{2}-1} s$ for $s \in U_{i}$ and $v$ is a product of words $\left(s s_{j}\right)^{\frac{m}{2}-1} s$ for $s \in U_{j}$.) What can $k$ and $l$ be? The following lemmas effectively guarantee that for any choice of $k$ and $l$ as above, there are numbers $k^{\prime}$ and $l^{\prime}$ such that an automorphism corresponding to these choices is inverse to the first. The proof of the first involves elementary number theory and is omitted. The proof of the second requires tedious but straightforward computation and will also be omitted.

Lemma 4.3. Let $k$ and $n$ be as above. Then there is a unique number $k^{\prime}, 1 \leq k^{\prime} \leq$ $n-1$, such that $\left(k^{\prime}, n\right)=1$ and $k k^{\prime}+1=d n$ for some integer $d$.

Lemma 4.4. Let $\gamma_{l}, u, v, k, k^{\prime}$, and $d$ be as above. Let $u^{\prime}=u^{-1}$ and $v^{\prime}=v^{-1}$. Let $\gamma_{l^{\prime}}^{\prime}$ be any map of $W$ satisfying:
1. $\gamma_{l^{\prime}}^{\prime}(s)=w_{i}^{\prime} s w_{i}^{\prime-1}$ for $s \in U_{i}$,
2. $\gamma_{l^{\prime}}^{\prime}(s)=w_{i}^{\prime} u^{\prime} x_{l^{\prime}}\left(k^{\prime}\right) v^{\prime} s v x_{l^{\prime}}\left(k^{\prime}\right)^{-1} u w_{i}^{\prime-1}$ for $s \in U_{j}$, and
3. $\gamma_{l^{\prime}}^{\prime}\left(w_{i}\right) w_{i}^{\prime}=1$. 
If $d$ is odd, then

$$
\gamma_{1} \circ \gamma_{4}^{\prime}(s)=\gamma_{4}^{\prime} \circ \gamma_{1}(s)=\gamma_{2} \circ \gamma_{3}^{\prime}(s)=\gamma_{3}^{\prime} \circ \gamma_{2}(s)=s
$$

for all $s \in U_{i} \cup U_{j}$. If $d$ is even, then

$$
\gamma_{l} \circ \gamma_{l}^{\prime}(s)=\gamma_{l}^{\prime} \circ \gamma_{l}(s)=s
$$

for all $s \in U_{i} \cup U_{j}$ and $l=1,2,3,4$.

Therefore, any choice of $x_{l}(k)$ yields a map which is "invertible" when restricted to $U_{i} \cup U_{j}$. We have $w_{j}=w_{i} u x_{l}(k) v$ for any $k$ such that $(k, n)=1$, any $l \in$ $\{1,2,3,4\}$, and any $u$ and $v$ as above. (Note that we include neither $\epsilon_{s_{i}} \in\left\{1, s_{i}\right\}$ nor $\epsilon_{s_{j}} \in\left\{1, s_{j}\right\}$ as was done before, as $s_{i} x_{l}(k)=x_{l^{\prime}}(n-k)$ for some $l^{\prime}$ and $x_{l}(k) s_{j}=x_{l^{\prime}}(k)$ for some $l^{\prime}$.)

Case 4. Now $\left|U_{i}\right|>1$ and $U_{j}=\left\{s_{j}\right\}$. As was argued in Section 3, there exists a unique vertex $s_{i} \in U_{i}$ such that $\left[s_{i} s_{j}\right]$ is an edge in $\mathcal{V}$, and this vertex $s_{i}$ is a junction separating $U_{i}$ and $U_{j}$. We can now show, arguing as before, that $w_{j}=w_{i} u x_{1}(k)$, where $u$ is as before, and we select the single element $x_{1}(k)$ from each set of four which elements conjugates $s_{j}$ to $\left(s_{j} s_{i}\right)^{k-1} s_{j}$. (Recall that the effect of conjugating $s_{j}$ by any one of these four elements is the same, for a given $k$.)

Case 5. Now $U_{i}=\left\{s_{i}\right\}$ and $\left|U_{j}\right|>1$. We can argue as above to show that $w_{j}=w_{i} u x_{l}(k) v$, where $u, v$, and $x_{l}(k)$ are defined as before. (Note that if $s_{i}$ has degree $1, u$ must be trivial.)

Case 6. Finally, suppose that $\left|U_{i}\right|=\left|U_{j}\right|=1$. In this case we may show that $w_{j}=w_{i} u x_{1}(k)$, where $u$ and $x_{1}(k)$ are as before. As in Case $5, u=1$ if $s_{i}$ has degree 1.

\section{The Unit GRAPH AND the STRUCtURE OF AN AUtOMORPHISM}

We now understand how to modify our conjugating element when passing from one unit to any adjacent unit. In this section we piece together this local information to obtain an arbitrary automorphism.

We define the unit graph $\Gamma=\Gamma(\mathcal{V})$ of the diagram $\mathcal{V}$ as the (unlabeled) graph whose vertex set is $\mathcal{U}$ and for which there is an edge $\left[U_{i} U_{j}\right]$ between units $U_{i}$ and $U_{j}$ whenever $U_{i}$ and $U_{j}$ are adjacent.

It is easy to see that $\Gamma$ is connected. (One can connect any two units by keeping track of the units that are entered in tracing a path from a vertex in one unit to a vertex in the other.) In $\Gamma$, choose any spanning tree $T$.

Let $\{s\}$ be any single-vertex junction in $\mathcal{V}$. Because $\mathcal{V}$ is NVB, we may divide the units of $\mathcal{V}$ which contain $s$ into two sets, depending the connected component of $\mathcal{V} \backslash\{s\}$ in which a given unit lies. Denote these two subsets of $\mathcal{U}$ by $\mathcal{U}_{1}(s)$ and $\mathcal{U}_{2}(s)$. We claim that we can modify the spanning tree $T$ to obtain a new spanning tree $T^{\prime}$ which has the property that any time $\{s\}$ is a single-vertex junction, there is exactly one pair $\left(U_{1}, U_{2}\right) \in \mathcal{U}_{1}(s) \times \mathcal{U}_{2}(s)$ for which $\left[U_{1} U_{2}\right]$ is an edge in $T^{\prime}$.

Indeed, let $s$ be as above. Clearly $T$ contains at least one edge as described above to begin with (this is because $\{s\}$ is a junction). Denote it by $\left[U_{1} U_{2}\right]$. By the definition of $\mathcal{U}_{i}(s)(i=1,2)$, it is clear that every element $U \in \mathcal{U}_{1}(s)$ is connected to $U_{1}$ in $\Gamma$, and similarly every element $U \in \mathcal{U}_{2}(s)$ is connected to $U_{2}$ in $\Gamma$. Therefore 
if $\left[U_{1}^{\prime} U_{2}^{\prime}\right]$ is any other edge in $T$ for $\left(U_{1}^{\prime}, U_{2}^{\prime}\right) \in \mathcal{U}_{1}(s) \times \mathcal{U}_{2}(s)$, we can eliminate this edge from $T$ and maintain connectivity by adding to $T$ (if necessary) paths from $U_{1}^{\prime}$ to $U_{1}$ and from $U_{2}^{\prime}$ to $U_{2}$. (Essentially, one simply creates a spanning tree for the subgraph $\mathcal{U}_{1}(s) \cup \mathcal{U}_{2}(s)$ which uses the prescribed edge $\left[U_{1} U_{2}\right]$.)

The reader should convince himself or herself that this modification can be performed independently for all single-vertex junctions. (This is so because no elements of $\mathcal{U}_{1}(s)$ are separated from each other by single-vertex junctions, by Lemma 4.2.)

Remark. Why perform this modification? We shall use the tree $T^{\prime}$ to define an automorphism by stepping from one vertex of $T^{\prime}$ to an adjacent vertex and modifying the conjugating word in a manner appropriate for the edge so traversed. Suppose $s \in U_{i} \cap U_{j}$ and $U_{i}, U_{j} \in \mathcal{U}_{1}(s)$. Then Lemma 4.2 implies that the conjugating words $w_{i}$ and $w_{j}$ must differ only by a product of words $\left(s s_{k}\right)^{\frac{m_{k}}{2}}$ for $s_{k}$ lying in the same connected component. In order that we yield such a modification, we must not be allowed to "cross over" a single-vertex junction and then "cross back".

Now pick and fix any vertex $U_{0}$ in $T^{\prime}$ such that $U_{0}$ is not a single-vertex unit $\{s\}$ for $s$ a vertex of degree 1. (It is clear that this choice can always be made.) Call $U_{0}$ the basepoint of $T^{\prime}$. Since $T^{\prime}$ is a tree, there is a unique geodesic (shortest) path from $U_{0}$ to any other vertex $U$ of $T^{\prime}$. We assign an orientation to the edges of $T^{\prime}$. If $\left[U_{i} U_{j}\right]$ is any edge in $T^{\prime}$, we define $U_{i}$ to be the initial point of $\left[U_{i} U_{j}\right]$ and $U_{j}$ to be the terminal point of $\left[U_{i} U_{j}\right]$ if the geodesic path from $U_{0}$ to $U_{j}$ passes through $U_{i}$.

We now label the (oriented) edges of $T^{\prime}$ with elements of $W$. For an edge $\left[U_{i} U_{j}\right]$, we will denote its label by $\phi_{\left[U_{i} U_{j}\right]}$.

The edges of $T^{\prime}$ are of six types, corresponding exactly to the six cases considered in the previous section. For any junction $J$ separating $U_{i} \backslash J$ and $U_{j} \backslash J$, let $A_{i}(J)$ be the connected component of $\mathcal{V} \backslash J$ containing $U_{i} \backslash J$, and let $A_{j}(J)$ be the connected component of $\mathcal{V} \backslash J$ containing $U_{j} \backslash J$.

1. If $\left|U_{i}\right|>1,\left|U_{j}\right|>1$, and $U_{i} \cap U_{j}=\left\{s_{1}, s_{2}\right\}$ is a junction separating $U_{i}$ and $U_{j}$, then $\phi_{\left[U_{i} U_{j}\right]}=u$, where $u \in\left\{1,\left(s_{1} s_{2}\right)^{\frac{m_{12}}{2}}\right\}$.

2. If $\left|U_{i}\right|>1,\left|U_{j}\right|>1$, and $U_{i} \cap U_{j}=\{s\}$ is a junction separating $U_{i}$ and $U_{j}$, then $\phi_{\left[U_{i} U_{j}\right]}=\epsilon_{s} u v$, where $\epsilon_{s} \in\{1, s\}, u$ is a product of words $\left(s_{i} s\right)^{\frac{m_{i}}{2}-1} s_{i}$ for $s_{i}$ in $A_{i}(\{s\})$, and $v$ is a product of words $\left(s_{j} s\right)^{\frac{m_{j}}{2}-1} s_{j}$ for $s_{i}$ in $A_{j}(\{s\})$.

3. If $\left|U_{i}\right|>1,\left|U_{j}\right|>1, U_{i} \cap U_{j}=\emptyset$, and $\left[s_{i} s_{j}\right]$ is an edge labeled $n$ for $s_{i} \in U_{i}$ and $s_{j} \in U_{j}$, then $\phi_{\left[U_{i} U_{j}\right]}=u x_{l}(k) v$, where $u$ is a product of words $\left(s s_{i}\right)^{\frac{m}{2}-1} s$ for $s \in A_{i}\left(\left\{s_{i}\right\}\right), v$ is a product of words $\left(s s_{j}\right)^{\frac{m}{2}-1} s$ for $s \in A_{j}\left(\left\{s_{j}\right\}\right)$, and $x_{l}(k)$ is one of the words in (4) from Section 4 , for some $k$ such that $(k, n)=1$ and some $l=1,2,3,4$.

4. If $\left|U_{i}\right|>1, U_{j}=\left\{s_{j}\right\}$, and $s_{i} \in U_{i}$ is such that $\left[s_{i} s_{j}\right]$ is an edge in $\mathcal{V}$ labeled $n$, then

a. if $s_{j}$ has degree $1, \phi_{\left[U_{i} U_{j}\right]}=u x_{1}(k)$, and

b. if $s_{j}$ has degree $2, \phi_{\left[U_{i} U_{j}\right]}=u x_{l}(k)$,

where $u$ is a product of words $\left(s s_{i}\right)^{\frac{m}{2}-1} s$ for $s \in A_{i}\left(\left\{s_{i}\right\}\right)$, and $x_{l}(k)$ is as in (4) from Section 4 , for some $k$ such that $(k, n)=1, l=1,2,3,4$.

5. If $U_{i}=\left\{s_{i}\right\},\left|U_{j}\right|>1$, and $s_{j} \in U_{j}$ is such that $\left[s_{i} s_{j}\right]$ is an edge in $\mathcal{V}$ labeled $n$, then $\phi_{\left[U_{i} U_{j}\right]}=x_{l}(k) v$, where $x_{l}(k)$ and $v$ are as in Case 3, where we allow only $l=1$ and $l=2$. 
6. If $U_{i}=\left\{s_{i}\right\}$ and $U_{j}=\left\{s_{j}\right\}$ and $\left[s_{i} s_{j}\right]$ is an edge labeled $n$ in $\mathcal{V}$, then

a. if $s_{j}$ has degree 1 , then $\phi_{\left[U_{i} U_{j}\right]}=x_{1}(k)$, and

b. if $s_{j}$ has degree 2, then $\phi_{\left[U_{i} U_{j}\right]}=x_{l}(k)$,

where $x_{l}(k)$ is as in Case 3, for either $l=1$ or $l=2$.

We note that the labels we give each type of edge differ only slightly from the corresponding ratios $w_{i}^{-1} w_{j}$ computed in the previous section. In order to explain the differences, we will need the following simple lemma, whose proof consists of direct computation.

Lemma 5.1. Let the word $x_{l}(k)$ be defined as above, relative to the edge $\left[s_{i} s_{j}\right]$ labeled $n$. Then:

a. $x_{3}(k)=s_{i} x_{1}(n-k)$ and $x_{4}(k)=s_{i} x_{2}(n-k)$ for all $k$ such that $(k, n)=1$, and

b. for all $k$ such that $(k, n)=1, l \in\{1,2,3,4\}$, and $z \in\left\{1, s_{j},\left(s_{i} s_{j}\right)^{\frac{n}{2}-1} s_{i}\right.$, $\left.\left(s_{i} s_{j}\right)^{\frac{n}{2}}\right\}$, there exists $l^{\prime} \in\{1,2,3,4\}$ such that $x_{l}(k) z=x_{l^{\prime}}(k)$.

In Case 5 , we seem to have lost generality by removing the term $u$ and by allowing only $l=1$ and $l=2$.

However, if $s_{i}$ is a vertex with degree $1, u=1$ must hold. When $s_{i}$ has degree 2, $u \in\left\{1, s_{i},\left(s s_{i}\right)^{\frac{m}{2}-1} s,\left(s s_{i}\right)^{\frac{m}{2}}\right\}$ (where $\left[s s_{i}\right]$ is an edge labeled $m, s \neq s_{j}$ ). Further, Lemma 5.1 allows us to factor a single letter $s_{i}$ out of either $x_{3}(k)$ or $x_{4}(k)$. This letter can then be multiplied with $u$; the resulting product may then be absorbed by the term $x_{l}(k)$ which occurs in the word $\phi_{\left[\{s\}\left\{s_{i}\right\}\right]}$. Lemma 5.1 now guarantees that such absorption does not alter the element to which $s_{i}$ is conjugated. (Also note that it is clear that the edge $\left[\{s\}\left\{s_{i}\right\}\right]$ must appear in $T^{\prime}$.)

In Case 6, we may omit the term $u$ for the same reason. In this case and in Case 4 , we must also be ready to absorb a term which may come from the following edge in $T^{\prime}$. We allow $l=1$ and $l=2$ in Case 6 to account for this absorption. If either $x_{3}(k)$ or $x_{4}(k)$ in this term arises as a result of absorption, we may factor out a single letter $s_{i}$ and push it into the previous edge. This process, analogous to "carrying" in arithmetic, must terminate when we reach a junction as in Case 4, where we allow any value of $l$ in $\{1,2,3,4\}$.

Remark. These precautions are taken in order for the description of an automorphism given below to be unique.

Finally, we label the vertex $U_{0}$ with any element $\phi_{0}$ of $W$.

Every such labeling defines an automorphism $\phi$ in the following fashion. Fix a labeling as described above and let $s$ be a generator in $S$, and choose any unit $U$ which contains $s$. Let $\left\{\left[U_{0} U_{1}\right],\left[U_{1} U_{2}\right], \ldots,\left[U_{l-1} U_{l}\right]\right\}$ be the unique geodesic in $T^{\prime}$ from $U_{0}$ to $U_{l}=U$. Then define

$$
\phi_{s}=\phi_{0} \prod_{i=0}^{l-1} \phi_{\left[U_{i} U_{i+1}\right]}
$$

and

$$
\phi(s)=\phi_{s} s \phi_{s}^{-1} .
$$

First note that the choice of the unit $U$ containing $s$ is not important. Indeed, suppose $s \in U_{i} \cap U_{j}$. Then $s$ is contained in a junction separating those two units and one easily sees that $\phi_{\left[U_{i} U_{j}\right]}$ must commute with $s$. 
By considering group relations, it is easy to see that this map is a homomorphism. To see that $\phi$ is an automorphism, we now indicate a formula for the composition of two such maps, and a formula for the inverse of a given map.

Let $\phi$ and $\phi^{\prime}$ be two homomorphisms defined as above. Let $\psi=\phi^{\prime} \circ \phi$. We compute $\psi$ by computing $\psi_{\left[U_{i} U_{j}\right]}$ for each edge $\left[U_{i} U_{j}\right]$, depending on the type of each edge:

1. $\psi_{\left[U_{i} U_{j}\right]}=\phi_{\left[U_{i} U_{j}\right]}^{\prime} \phi_{\left[U_{i} U_{j}\right]}$.

2. If $\phi_{\left[U_{i} U_{j}\right]}=\epsilon_{s} u v$ and $\phi_{\left.U_{i} U_{j}\right]}^{\prime}=\epsilon_{s}^{\prime} u^{\prime} v^{\prime}$, then $\psi_{\left[U_{i} U_{j}\right]}=\epsilon_{s} \epsilon_{s}^{\prime} u u^{\prime} v^{\prime} v$.

3. If $\phi_{\left[U_{i} U_{j}\right]}=u x_{l}(k) v$ and $\phi_{\left[U_{i} U_{j}\right]}^{\prime}=u^{\prime} x_{l^{\prime}}\left(k^{\prime}\right) v^{\prime}$, then $\psi_{\left[U_{i} U_{j}\right]}=u u^{\prime} x_{l \circ l^{\prime}}\left(k k^{\prime}\right) v^{\prime} v$, where $\{1,2,3,4\}$ is made a group isomorphic to the 4-element group $V$ with 1 as its unit and product $\circ$.

4. If $\phi_{\left[U_{i} U_{j}\right]}=u x_{l}(k)$ and $\phi_{\left[U_{i} U_{j}\right]}^{\prime}=u^{\prime} x_{l^{\prime}}\left(k^{\prime}\right)$, then $\psi_{\left[U_{i} U_{j}\right]}=u u^{\prime} x_{l \circ l^{\prime}}\left(k k^{\prime}\right)$, with $l \circ l^{\prime}$ defined as in Case 3 .

5 . If $\phi_{\left[U_{i} U_{j}\right]}=x_{l}(k) v$ and $\phi_{\left[U_{i} U_{j}\right]}^{\prime}=x_{l^{\prime}}\left(k^{\prime}\right) v^{\prime}$, then $\psi_{\left[U_{i} U_{j}\right]}=x_{l \circ l^{\prime}}\left(k k^{\prime}\right) v^{\prime} v$, with $l \circ l^{\prime}$ defined as in Case 3 .

6. If $\phi_{\left[U_{i} U_{j}\right]}=x_{l}(k)$ and $\phi_{\left[U_{i} U_{j}\right]}^{\prime}=x_{l^{\prime}}\left(k^{\prime}\right)$, then $\psi_{\left[U_{i} U_{j}\right]}=x_{l \circ l^{\prime}}\left(k k^{\prime}\right)$, with $l \circ l^{\prime}$ defined as in Case 3.

Finally, given $\phi_{0}$ and $\phi_{0}^{\prime}$ corresponding to $\phi$ and $\phi^{\prime}$, we compute $\psi_{0}=\phi^{\prime}\left(\phi_{0}\right) \phi_{0}^{\prime}$. This completes the description of the composition $\psi$.

Using Lemma 4.4 in order to compute "inverses" for the terms $x_{l}(k)$, and letting $u^{\prime}=u^{-1}$ and $v^{\prime}=v^{-1}$ throughout, it is now easy to compute the inverse $\phi^{-1}$ of a given map $\phi$. (The most difficult part is to "invert" $\phi_{0}$.) Therefore, every map so constructed is an automorphism of $W$.

It is clear by the arguments given in Section 4 that all automorphisms $\phi$ which satisfy $\phi(s)=w_{s} s w_{s}^{-1}$ for all $s \in S$ (where $w_{s}$ depends on $s$ ) can be obtained in this fashion. Moreover it is not difficult to show that if $\phi$ and $\phi^{\prime}$ are defined as above, then $\phi=\phi^{\prime}$ if and only if $\phi_{0}=\phi_{0}^{\prime}$ and $\phi_{\left[U_{i} U_{j}\right]}=\phi_{\left[U_{i} U_{j}\right]}^{\prime}$ for all edges $\left[U_{i} U_{j}\right]$ in $T^{\prime}$.

Given any automorphism of $W$, we obtained an automorphism in the collection $G$ of automorphisms described above by composing it with an element of $\operatorname{Diag}(W)$. The intersection $G \cap \operatorname{Diag}(W)$ is trivial, so that every element of $\operatorname{Aut}(W)$ can be written uniquely as such a composition. Thus $\operatorname{Aut}(W)$ is a semidirect product of $G$ by $\operatorname{Diag}(W)$, and we have proved the main theorem.

It is easy to see that the inner automorphisms and diagram automorphisms, along with automorphisms for which $\phi_{\left[U_{i} U_{j}\right]}=1$ for all but a single edge $\left[U_{i} U_{j}\right]$ in $T^{\prime}$, generate $\operatorname{Aut}(W)$. Thus $\operatorname{Aut}(W)$ is finitely generated. It is not difficult to explicitly describe a finite presentation for $\operatorname{Aut}(W)$ in terms of these generators, given the formula for composition given above.

The corollaries given in the Introduction are easily proven. Because $|\operatorname{Diag}(W)|<$ $\infty$, the only way in which $\operatorname{Out}(W)$ can be infinite is if there are infinitely many choices for $\phi_{\left[U_{i} U_{j}\right]}$, for some edge $\left[U_{i} U_{j}\right]$ in $T^{\prime}$. This is clearly the case only when there is a cut vertex which is adjacent to more than two vertices in $\mathcal{V}$. Thus Corollary 1.2 follows.

In fact, consider the subgroup $F$ of $\operatorname{Aut}(W)$ generated by those $\phi$ for which $\phi_{\left[U_{i} U_{j}\right]}=1$ for edges of types 1 and 6 , and $\epsilon_{s}=1$ and $x_{l}(k)=1$ for all occurrences of these words in edges of types $2,3,4$, and 5 . (The group $F$ has finite index in Out $(W)$.) Then $F$ is clearly generated by a set of involutions whose cardinality 
can be read immediately from $\mathcal{V}$ by counting the number of vertices adjacent to cut vertices. It is not difficult to see that $F$ is a direct product of free powers of $Z_{2}$, proving Corollary 1.4.

It is also easy to compute the order of $\operatorname{Out}(W)$ if this group is finite. If there is a cut vertex, Corollary 1.2 and the fact that $\mathcal{V}$ is NVB imply that $\mathcal{V}$ must be a path of length $r$ which does not intersect itself. Let $n_{i}$ be the label on the $i$ th edge, proceeding from one of the endpoints of this path. Then $|\operatorname{Out}(W)|=\delta n$, where

$$
n=2^{r-2} \prod_{i=1}^{r} \phi\left(n_{i}\right)
$$

( $\phi$ is Euler's totient function), and $\delta \in\{1,2\}$ (depending on whether the path $\mathcal{V}$ has one or two symmetries).

If there are no cut vertices, then the only junctions are edges. If $k$ is the number of units into which $\mathcal{V}$ is divided by these edges, it is easy to see that $\operatorname{Aut}(W)$ has the structure given in Corollary 1.3, and $|\operatorname{Out}(W)|=2^{k}|\operatorname{Diag}(W)|$.

Remark. The above discussion completes the proof of all results stated in the case where $\mathcal{V}$ has no spikes (i.e., is rigid). If such spikes [st] do exist, it is easy to show that there are only finitely many diagrams $\mathcal{V}^{\prime}$ corresponding to $(W, S)$, each of which can be obtained from $\mathcal{V}$ by "expanding" spikes [st] (labeled $2(2 k+1)$ ) to triangles $\left\{s_{1}, s_{2}, s_{3}\right\}$ with edge multiset $\{2,2,2 k+1\}$. Each such diagram $\mathcal{V}^{\prime}$ comes equipped with an automorphism of $W$ which takes the original generating set to the new generating set in the necessary fashion. These new maps, along with those described above, generate $\operatorname{Aut}(W)$, and clearly the subgroup of $\operatorname{Aut}(W)$ described above has finite index in $\operatorname{Aut}(W)$. Thus even in this case all of the results stated in the first section hold, as we are concerned only with behavior up to finite index.

\section{Free products}

As promised in the Introduction, let us briefly consider the issue of free products of Coxeter groups. Any Coxeter group can easily be written as a free product of freely indecomposable Coxeter groups. Indeed, a Coxeter group is freely indecomposable if and only if its diagram is connected. Moreover, given two Coxeter groups $W_{1}$ and $W_{2}$ with diagrams $\mathcal{V}_{1}$ and $\mathcal{V}_{2}$, respectively, a diagram for $W_{1} * W_{2}$ is obtained by taking the the disjoint union of $\mathcal{V}_{1}$ and $\mathcal{V}_{2}$.

First let us consider the case of a Coxeter group $W$ which decomposes as a free product of exactly two freely indecomposable Coxeter groups (thus its diagram $\mathcal{V}$ has exactly two connected components). We write $W \cong W_{1} * W_{2}$ for some Coxeter groups $W_{i}$ with diagrams $\mathcal{V}_{i} \subseteq \mathcal{V}, i=1,2$, whose disjoint union is $\mathcal{V}$.

Let us assume that $W$ is a large-type even rigid Coxeter group, and that both $W_{1}$ and $W_{2}$ are strongly rigid. (It is easy to see that $W$ itself cannot be strongly rigid.) Consider any automorphism $\phi$ of $W$ such that for every $s$ there is an element $w \in W$ such that $\phi(s)=w s w^{-1}$. (As in Section 4 , any $\phi \in \operatorname{Aut}(W)$ can be composed with a diagram automorphism to yield such an automorphism.)

By strong rigidity there are elements $w_{i} \in W_{i}$ such that $\phi\left(s_{i}\right)=w_{i} s_{i} w_{i}^{-1}$ for $s_{i} \in W_{i} \cap S, i=1,2$. In order to completely describe $\phi$, we need only compute the ratio $w_{1}^{-1} w_{2}$. An argument almost identical to the proof of Lemma 4.1 yields the following result. 
Lemma 6.1. Let $W, W_{i}$, and $w_{i}$ be as above. Then $w_{1}^{-1} w_{2}=u_{1} u_{2}$, where $u_{i} \in W_{i}$ for $i=1,2$.

Therefore every automorphism which takes a generator $s$ to a conjugate of $s$ is described by a triple $\left(w, u_{1}, u_{2}\right)$. If $\phi$ is given by $\left(w, u_{1}, u_{2}\right)$ and $\phi^{\prime}$ by $\left(w^{\prime}, u_{1}^{\prime}, u_{2}^{\prime}\right)$, it is easy to show that composition is given by the formula

$$
\left(w^{\prime}, u_{1}^{\prime}, u_{2}^{\prime}\right) \circ\left(w, u_{1}, u_{2}\right)=\left(\phi^{\prime}(w) w^{\prime}, u_{1} u_{1}^{\prime}, u_{2}^{\prime} u_{2}\right) .
$$

From this formula inverses can be easily computed. If neither $W_{1}$ nor $W_{2}$ is finite, two distinct triples correspond to distinct automorphisms. (In general, one must perform a further quotient by the centralizers $C\left(W_{i}\right)$ for $i=1,2$, but $C\left(W_{i}\right)$ is trivial if $W_{i}$ is infinite.) We obtain the following result.

Theorem 6.2. Let $W$ be a large-type even rigid Coxeter group which decomposes as the free product of exactly two strongly rigid Coxeter groups $W_{1}$ and $W_{2}$. Then

$$
\operatorname{Aut}(W) \cong G \times \operatorname{Diag}(W),
$$

where $G$ is (element-wise) a product of $W, W_{1}$, and $W_{2}$, with multiplication given above. Also, Out $(W)$ is finite if and only if both $W_{1}$ and $W_{2}$ are finite; that is, both are isomorphic either to $Z_{2}$ or to a strongly rigid dihedral group $D_{n}$, for some $n$.

The statement regarding finiteness of $\operatorname{Out}(W)$ is clear.

The preceding arguments can also be used in a much more general setting in order to establish the following result.

Theorem 6.3. Let $G \cong G_{1} * G_{1}$ where Out $\left(G_{i}\right)$ is finite for $i=1,2$. Then $\operatorname{Aut}(G)$ contains a subgroup of finite index which is isomorphic to a product of $G, G_{1}$, and $G_{2}$, with composition given by

$$
\phi^{\prime} \circ \phi=\left(g^{\prime}, g_{1}^{\prime}, g_{2}^{\prime}\right) \circ\left(g, g_{1}, g_{2}\right)=\left(\phi^{\prime}(g) g^{\prime}, g_{1} g_{1}^{\prime}, g_{2}^{\prime} g_{2}\right)
$$

for $g \in G$ and $g_{i} \in G_{i}, i=1,2$.

To finish, let us now consider a Coxeter group $W$ which decomposes as a free product of finitely many freely indecomposable Coxeter groups: $W=W_{1} * \cdots * W_{k}$. Let us furthermore assume that each $W_{i}(i=1, \ldots, k)$ is even, of large type, and is NVB. Each $W_{i}$ is finitely presented, and the results from Section 5 guarantee that $\operatorname{Aut}(W)$ is finite presented for every $i$. Therefore, we appeal to [9, which produces an explicit (and finite!) presentation for the group $\operatorname{Aut}(W)$. We obtain the following

Theorem 6.4. Let $W$ be the free product of the even, large-type, NVB Coxeter groups $W_{i}$. Then $\operatorname{Aut}(W)$ is finitely presented. (Moreover, one may explicitly compute a presentation.)

If $k \geq 3$, Out $(W)$ will always be infinite.

\section{REFERENCES}

1. Bahls, P., "Even rigidity in Coxeter groups", Ph.D. Thesis, Vanderbilt University, 2002.

2. Bahls, P., "Strongly rigid even Coxeter groups", Topology Proceedings 28 (2004) no. 1, 19-54. MR.2105446 (2005i:20063)

3. Bahls, P., and Mihalik, M., "Reflection independence in even Coxeter groups", Geom. Ded. 110 (2005) no. 1, 63-80. MR 2136020

4. Bahls, P., and Mihalik, M., "Centralizers of parabolic subgroups of Coxeter groups", preprint, 2003. 
5. Bourbaki, N., Groupes et Algebres de Lie, Chap. IV-VI, Hermann, Paris, 1981. MR0647314 (83g:17001)

6. Charney, R., and Davis, M., "When is a Coxeter group determined by its system?", J. London Math. Soc. (2) 61 (2000) no. 2, 441-461. MR.1760693 (2001i:20078)

7. Franzsen, W., and Howlett, R., "Automorphisms of Coxeter groups of rank 3", Proc. Am. Math. Soc. 129 (2001) no. 9, 2607-2616. MR1838783 (2002c:20060)

8. Franzsen, W., "Automorphisms of Coxeter groups of rank 3 with infinite bonds," J. Algebra 248 (2002) no. 1, 381-396. MR1879023 (2003f:20060)

9. Gilbert, N., "Presentations of the automorphism group of a free product," Proc. London Math. Soc. (3) 54 (1987), 115-140. MR0872253 (87m:20076)

10. Howlett, R., Rowley, P., and Taylor, D., "On outer automorphism groups of Coxeter groups", Manuscripta Math. 93 (1997) no. 4, 499-513. MR1465894 (98j:20056)

11. James, L., "Complexes and Coxeter groups - operations and outer automorphisms", J. Algebra 113 (1988) no. 2, 339-345. MR0929764 (89c:20055)

12. Levitt, G., "Automorphisms of hyperbolic groups and graphs of groups", preprint, 2003.

13. Lyndon, R., and Schupp, P., Combinatorial Group Theory, Ergebnisse series, vol. 89, Springer, New York, 1977 (reprinted in the Springer Classics in Mathematics Series, 2000). MR 1812024 (2001i:20064)

14. Mihalik, M., and Tschantz, S., "Visual decompositions of Coxeter groups", preprint, 2001.

15. Mühlherr, B., "Automorphisms of graph-universal Coxeter groups", J. Algebra 200 (1998) no. 2, 629-649. MR:1610676 (98m:20048)

16. Mühlherr, B., and Weidmann, R., "Rigidity of skew-angled Coxeter groups", Adv. Geom. 2 (2002) no. 4, 391-415. MR.1941338 (2003h:20073)

17. Rips, E., and Sela, Z., "Structure and rigidity in hyperbolic groups I", Geom. Func. Anal. 4 no. 3 (1994), 337-371. MR.1274119 (96c:20067)

18. Tits, J., "Sur le groupe des automorphismes des certains groupes de Coxeter", J. Algebra 113 (1988) no. 2, 346-357. MR0929765 (89b:20077)

Department of Mathematics, University of Illinois at Urbana-Champaign, Urbana, ILLINOIS 61801

E-mail address: pbahls@math.uiuc.edu 